\title{
An atypical hydronephrosis revealing urinary tract involvement in antiphospholipid syndrome
}

Claire de Moreuil, ${ }^{\text {** }}$ Anne-Marie Piette, ${ }^{\mathrm{b}}$ Jean-Marie Hervé, ${ }^{\mathrm{c}}$ Philippe Camparo, ${ }^{\mathrm{d}}$

Anne-Catherine Baglin, ${ }^{\mathrm{d}}$ Olivier Blétry, ${ }^{\mathrm{b}}$ Jean-Emmanuel Kahn ${ }^{\mathrm{b}}$

a Service de Médecine interne, CHRU de Brest, Hôpital La Cavale Blanche, Boulevard Tanguy Prigent, 29609 Brest Cedex, France

${ }^{b}$ Service de Médecine interne, Hôpital Foch, 40 rue Worth, 92151, Suresnes, France

' Service d'Urologie, Hôpital Foch, 40 rue Worth, 92151, Suresnes, France

${ }^{d}$ Service d'Anatomo-Pathologie, Hôpital Foch, 40 rue Worth, 92151, Suresnes, France

\author{
Accepted on \\ June 29th, 2013 \\ DOI Name \\ http://dx.doi.org/10.3126/jaim.v2i2.7659 \\ Keywords \\ Antiphospholipid syndrome \\ Thrombosis \\ Hydronephrosis \\ Acute renal failure.

\section{Citation} \\ Claire de Moreuil, Anne-Marie Piette, Jean- \\ Marie Hervé, Philippe Camparo, Anne-Catherine \\ Baglin, Olivier Blétry, Jean-Emmanuel Kahn. An \\ atypical hydronephrosis revealing urinary tract \\ involvement in antiphospholipid syndrome. \\ Journal of Advances in Internal Medicine \\ 2013;02(02):78-80.
}

\begin{abstract}
Urinary tract involvement is rarely described in antiphospholipid syndrome (APS). We report the case of a 40 year-old man with primitive APS who developed an arterial and venous unilateral ureteral ischemia revealed by painful unilateral hydronephrosis. The patient was on oral anticoagulation for six years because of two deep venous thromboses. He developed then a massive splenic infarct and an acute myocardial infarct, which required the intensification of his anticoagulation and high doses of corticosteroids. Three months later, he was explored for a latero-thoracic pain. Biology found an acute renal failure and microscopic haematuria. CT scan showed a hypoperfusion of the left kidney, an ostial defect on renal artery and a left hydronephrosis. Retrograde pyelography found a stenosis of the proximal ureter. A dilatation with stenting failed, leading to a partial left ureteral resection. Histology confirmed ureteral segmental organized arterial thrombosis and venous thrombosis. Evolution was favourable with stabilization of creatinine.
\end{abstract}

\section{INTRODUCTION}

Antiphospholipid syndrome (APS) is a quite frequent etiology of acquired thrombophilia. It is characterized by venous or arterial thromboses, fetal losses and thrombocytopenia, in the presence of antiphospholipid antibodies (aPL) (lupus anticoagulant, anticardiolipin antibodies and/or anti-b2GP1). ${ }^{1}$ It can be associated with either auto-immune diseases such as systemic lupus erythematosus (SLE) or neoplasms, infections or drugs. Clinical manifestations are various. Deep venous thrombosis is the most frequently reported manifestation. ${ }^{2}$ Arterial thromboses are frequently located into the brain and can be associated with venous thromboses. Obstetrical complications include fetal losses, premature births and preeclampsia. Catastrophic APS (CAPS) is a rare $(<1 \%)$ but lifethreatening presentation, characterized by multiple small vessel thromboses that can lead to multi-organ failure. ${ }^{3}$

Our patient was diagnosed an atypical left hydronephrosis associated with acute renal failure while he was treated with oral vitamin $\mathrm{K}$ antagonists (VKA) and aspirin. The final diagnosis, histologically proven, was a left ureteral ischemia due to distal arterial and venous thromboses, associated with glomeruli lesions. To our knowledge, it is the first description of such a thrombosis localisation in APS.

\section{Case report}

Our patient was diagnosed on December 2001 a primitive APS after a first ilio-femoral left venous thrombosis. He was consuming tobacco up to one pack/day. Thrombophilia screenings found a lupus anticoagulant, with a Rosner index of 31. An oral anticoagulation (fluindione) was started but the patient wasn't compliant to INR controls. On December 2002, he developed a splenic infarct, without macroscopic thrombosis, while the INR was at 2,8. Aspirin was then added and the INR target was raised to $3-4$. He was still non-compliant

\footnotetext{
* Corresponding author

Dr Claire de Moreuil

Service de Médecine interne, CHRU de Brest,

Hôpital La Cavale Blanche, Boulevard Tanguy Prigent

29609 Brest Cedex. France

e-mail: claire.demoreuil@chu-brest.fr
} 
to treatment. On September 2008, he was hospitalized for an abdominal pain. CT scan showed several splenic infarcts with distal thrombosis of splenic artery, peri-splenic fat infiltration and left peri-ureteral infiltration. A few days later, he had an acute myocardial infarct with troponin elevation up to 35 $\mathrm{mg} / \mathrm{l}$, a subnormal coronarography and a cardiac MRI showing a necrosis in the infero-septal territory. Clinical examination found subungual flame-shaped hemorrhages consistent with digital microthrombi. Treatment was intensified with corticosteroids pulses, intravenous unfractionated heparin, aspirin, clopidogrel, b-blockers and ACE inhibitor. Evolution was positive and the patient left hospital one month later with VKA (INR target of 3-4), two platelet antiaggregants and prednisolone $10 \mathrm{mg} /$ day. He stopped smoking and became compliant to treatment and monitoring.

Three months later, on January 2009, the patient was rehospitalized for exploration of a recent left latero-thoracic pain. ECG, Chest X-Ray and echocardiography were normal. Blood tests found an increase in creatinine $(119 \mathrm{mmol} / \mathrm{l}$ against $69 \mathrm{mmol} / \mathrm{l}$ on September). INR was 4,2 and other parameters (Cell count, Troponin, CRP, D-Dimers) were normal. Urine tests showed a sample proteinuria of $0,24 \mathrm{~g} / \mathrm{l}$ and microscopic haematuria. A CT scan with contrast was then performed: a $15 \mathrm{~mm}$ left hilar adenopathy and a few little mediastinal adenopathies were found on the thoracic level. Fortuitously, a global hypoperfusion of the left kidney was noticed, with left pyelo-caliceal dilatation, left peri-ureteral and peri-renal fat infiltration (figure 1).

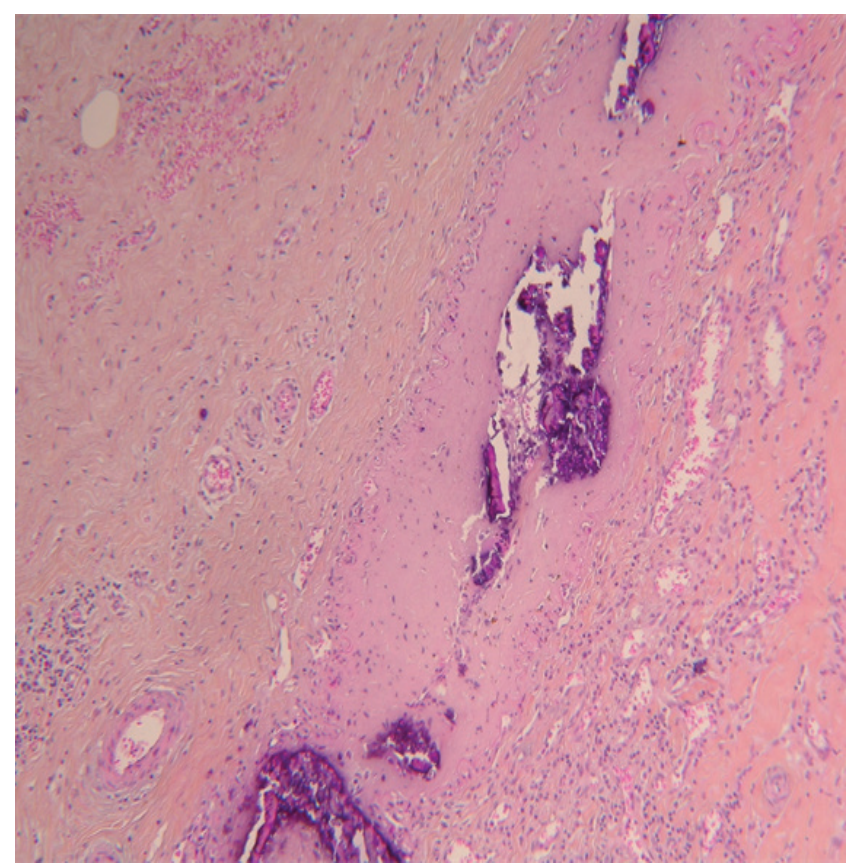

Figure 1. Abdominal CT scan. A. September 2008 (without contrast): left pyelo-caliceal dilatation. B. January 2009 (with contrast): left kidney hypoperfusion.

A retrograde ureteropyelography was then performed, showing a tight stenosis of the left proximal ureter on four $\mathrm{cm}$. It was impossible to insert an endoureteral prosthesis. Finally, the patient underwent a surgery: the pathologic ureteral portion was sectioned and an anastomosis was performed. Macroscopically, the ureteral tissue was fibrous and calcified.
Microscopically, there were peri-vascular inflammatory changes, thrombotic and calcified arteries and veins, inside fibro-collagen tissue, and glomerular lesions (figure 2).

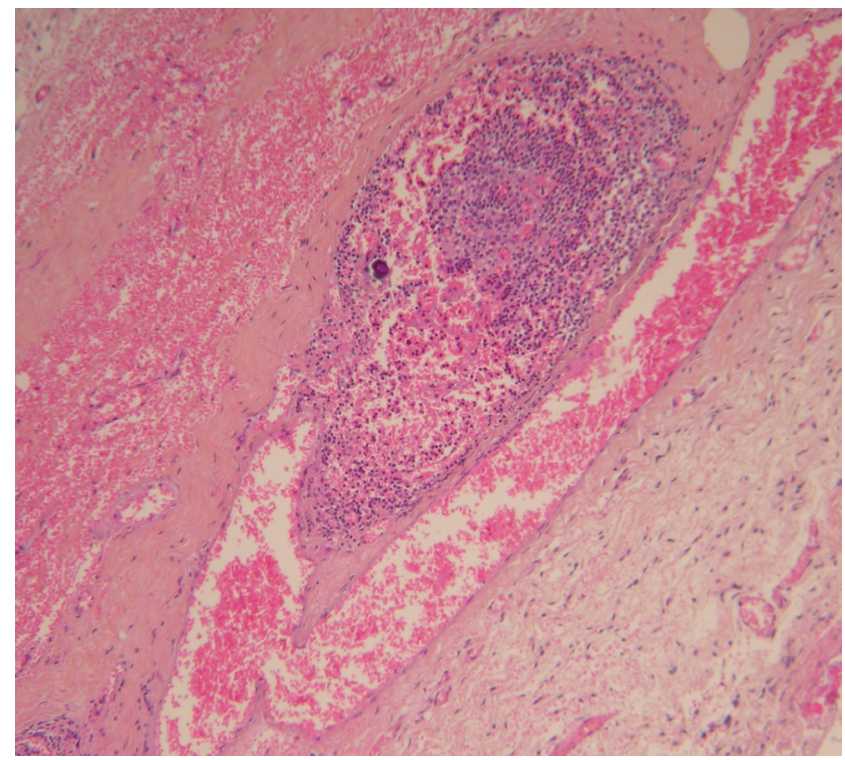

Figure 2. Histological findings. A. Ureteral section with venous thrombosis. B. Ureteral section with arterial thrombosis.

We conclude that it was a semi-recent both arterial and venous ureteral thrombosis, which had probably happened on September 2008 and hadn't been diagnosed then.

The glomerular lesions associated with acute renal failure, which was persistent despite urine surgical derivation, were considered as the proof of microvascular involvement. The patient was discharged after a few days, with maintenance of the same anticoagulant treatment. Renal function stabilized with a creatinine of $115 \mu \mathrm{mol} / \mathrm{I}$ (Cockroft-Gault clearance $=80$ $\mathrm{ml} / \mathrm{min})$.

\section{Discussion}

Urinary tract involvement is rarely described in APS. In SLE, chronic interstitial cystitis and secondary ureteral stenosis have occasionally been described, mostly associated with gastrointestinal symptoms (lupus enteropathy). ${ }^{4}$ We present here the first case of arterial and venous unilateral ureteral ischemia presenting as a hydronephrosis in a patient with APS.

Renal lesions include thrombotic microangiopathy lesions, which can be acute or chronic, and affect intra-parenchymatous arterioles and glomerular capillaries. Renal artery thrombosis is less frequent and can lead to renal infarct or reno-vascular hypertension. Our patient developed an acute renal failure without hypertension, probably due to minimal renal thrombotic microangiopathy.

CAPS or Asherson's syndrome is the worst manifestation of APS. Its mortality rate is up to $40 \%{ }^{5}$ It is defined as three or more simultaneous new thromboses in different organs/systems/ tissues, with histological confirmation of microvascular involvement and aPL detection. Our patient presented a CAPS, histologically proven, successfully managed with high-dose corticosteroids and intensification of anticoagulation. 
It has been proven that lupus anticoagulant was the biological test for APL detection the most associated with venous thromboses (risk ratio of 6 versus 2 with the other APL antibodies). ${ }^{6}$ Our patient had a lupus anticoagulant.

Treatment of thrombotic manifestations in APS is based on anticoagulation and platelet antiaggregants. There is a consensus to treat a first venous thrombosis in patients with APS with prolonged oral anticoagulation and an INR between 2 and 3. If thrombosis recurs under VKA or if it is an arterial thrombosis, the target INR is commonly risen to > 3 and antiaggregants are added.

CAPS is treated with high doses of intravenous unfractionned heparin and corticosteroids. In the life-threatening cases, plasmatic exchanges or intravenous immunoglobulins are added to lower plasmatic aPL rate. Indeed, it has been demonstrated that aPL were indirectly pathogenic by binding to phospholipid-binding plasma b2 glycoprotein I. Anti-domain I antibodies seem to be the most uniformly correlated with occurrence of thrombosis. ${ }^{7}$ Research on anti-I antibodies biological properties have found numerous ways of action on haemostasis: antibodies increase resistance against annexin $5,{ }^{8}$ then countering its anticoagulant properties; they inhibit the anticoagulant properties of protein $C,{ }^{9}$ independently of Factor $\mathrm{V}$ Leiden, and they increase concentration of activated Von Willebrand Factor. LDL receptors also have a crucial role in generating thrombosis: it was shown that antibodybound b2GP1 could bind and activate platelets through LDLreceptor-related protein. ${ }^{10}$ Moreover, APS is now described as an inflammatory pro-thrombotic disorder, as aPL are able to activate cellular elements (platelets, endothelial cells and monocytes), inhibit the fibrinolytic system, activate the coagulation cascade and activate the complement system. ${ }^{11}$

Our patient developed arterial and venous thromboses while he was on oral anticoagulation and platelet antiaggregants, what means he had a resistant APS. Rituximab (RTX) could be an effective treatment for resistant APS. Rubenstein et al ${ }^{12}$ published good results with RTX on three cases of APS, one of them with CAPS. It could be an alternative treatment for our patient if a new thrombosis occurs.

7. de Laat B, Mertens K, de Groot PG. Mechanisms of disease: antiphospholipid antibodies-from clinical association to pathologic mechanism. Nature clinical practice Rheumatology. 2008 Apr;4(4):192-9.

8. Rand $\mathrm{JH}, \mathrm{Wu} \mathrm{XX}$, Andree $\mathrm{HA}$, et al. Pregnancy loss in the antiphospholipid-antibody syndrome--a possible thrombogenic mechanism. The New England journal of medicine. 1997 Jul 17;337(3):154-60.

9. Gardiner $\mathrm{C}$, Cohen $\mathrm{H}$, Jenkins $\mathrm{A}$, et al. Detection of acquired resistance to activated protein $C$ associated with antiphospholipid antibodies using a novel clotting assay. Blood coagulation \& fibrinolysis : an international journal in haemostasis and thrombosis. 2006 Sep;17(6):477-83.

10. van Lummel $M$, Pennings $M T$, Derksen $R H$, et al. The binding site in \{beta\}2-glycoprotein I for ApoER2' on platelets is located in domain $\mathrm{V}$. The Journal of biological chemistry. 2005 Nov 4;280(44):36729-36.

11. Mehdi AA, Uthman I, Khamashta M. Antiphospholipid syndrome: pathogenesis and a window of treatment opportunities in the future. European journal of clinical investigation. 2010 May;40(5):451-64.

12. Rubenstein E, Arkfeld DG, Metyas S, et al. Rituximab treatment for resistant antiphospholipid syndrome. The Journal of rheumatology. 2006 Feb;33(2):355-7. 